\title{
INTERAÇÃO GENÓTIPOS X AMBIENTES PARA PRODUTIVIDADE DE CLONES DE Eucalyptus L'Hér. NO ESTADO DO RIO GRANDE DO SUL ${ }^{1}$
}

\author{
Gleison Augusto Santos ${ }^{2}$, Marcos Deon Vilela Resende ${ }^{3}$, Luciana Duque Silva ${ }^{4}$, Antonio Higa ${ }^{5}$ e \\ Teotônio Francisco Assis ${ }^{6}$
}

\begin{abstract}
RESUMO - Este trabalho objetivou estudar a interação genótipos em ambientes de clones de Eucalyptus em quatro ambientes do Estado do Rio Grande do Sul. Os ensaios foram conduzidos nas áreas da empresa CMPC Celulose Riograndense, nos Municípios de Minas do Leão (Horto Florestal Cambará), Encruzilhada do Sul (Horto Florestal Capivara), Dom Feliciano (Horto Florestal Fortaleza) e Vila Nova do Sul (Horto Florestal São João). No ano 2007, foi implantada uma rede de testes clonais com 864 clones de Eucalyptus, pertencentes a 37 espécies e híbridos. Aos 3 anos de idade, foram mensurados o diâmetro à altura do peito (dap) e a altura total (Ht) das árvores dos experimentos. O incremento médio anual (IMA) foi calculado de acordo com o volume individual por clone e o estande de plantas por hectare na idade de avaliação do teste clonal. Os resultados da correlação genotípica entre os ambientes variaram de 0,39 a 0,56 , demonstrando que ocorreu interação genótipos x ambientes significativa dos caracteres avaliados. Esses resultados indicam que é necessário selecionar clones específicos, nas combinações de ambientes com baixa correlação genética entre si. Ocorreu baixa correção genética entre os ambientes Cambará x Capivara; Cambará x São João; Capivara x Fortaleza; Capivara x São João; e Fortaleza x São João. Dessa maneira, para este estudo foram necessárias três unidades de manejo para minimizar os efeitos da interação genótipos x ambientes. Incluindo a seleção por estabilidade, adaptabilidade e os dois atributos simultaneamente, ocorreu mudança de clones e de posição entre os clones no ordenamento de seleção. Com a seleção simultânea por estabilidade e adaptabilidade (MHPRVG), os ganhos aumentaram em comparação com a seleção pelos valores genotípicos preditos entre os ambientes, principalmente, e houve incremento dos ganhos em relação à testemunha comercial. Por exemplo, os cinco melhores clones pelo critério MHPRVG apresentaram-se 56\% superiores à testemunha comercial para a característica produtividade em volume.
\end{abstract}

Palavras-chave: Adaptabilidade; Estabilidade; Ganho Genético.

\section{GENOTYPES X ENVIRONMENTS INTERACTION FOR PRODUCTIVITY OF Eucalyptus L'Hér. CLONES IN THE STATE OF RIO GRANDE DO SUL, BRAZIL}

\begin{abstract}
The present study aimed to study the genotypes interaction in environments of Eucalyptus clones, in four environments in the state of Rio Grande do Sul. The tests were conducted in the areas of company CMPC Celulose Riograndense, in the municipalities of Minas do Leão (farm Cambará), Encruzilhada do Sul (farm Capivara), Dom Feliciano (farm Fortaleza) and Vila Nova do Sul (farm São João). In 2007, a network of clonal tests was implanted with 864 clones of Eucalyptus, belonging to 37 different species and hybrids. At three years of age, we measured the diameter at breast height (dbh) and total height (Ht) of the trees of the experiments. The mean annual increment (MAI) was calculated according to the individual
\end{abstract}

\footnotetext{
${ }^{1}$ Recebido em 01.05.2013 aceito para publicação em 07.08.2014.

${ }_{2}^{2}$ CMPC Celulose Riograndense, Guaíba, RS - Brasil. E-mail: <gaugusto@cmpcrs.com.br>.

${ }^{3}$ Empresa Brasileira de Pesquisa Agropecuária, Centro Nacional de Pesquisa de Florestas, Colombo, PR - Brasil. E-mail: $<$ marcos.deon@gmail.com>.

${ }^{4}$ Universidade de São Paulo, Campus Luiz de Queiroz, Piracicaba, SP - Brasil. E-mail: <lucianaduques@yahoo.com.br>.

${ }^{5}$ Universidade Federal do Paraná, Setor de Ciências Agrárias, Departamento de Ciências Florestais, Curitiba, PR - Brasil. E-mail:<antonio.higa@gmail.com>.

${ }^{6}$ Assistech Tecnologia em Melhoramento, Nova Lima, MG - Brasil. E-mail: <assisteo@terra.com.br>.
} 
volume per clone and plant stand in hectare at age of evaluation of clonal test. The results for the genotypic correlations among environments ranged from 0.39 to 0.56 , showing that there was significant genotype $x$ environment interaction for all the evaluated characteristics. These results indicate that it is necessary to select specific clones for combinations of environments with low genetic correlation between them. Low genetic correlation occurred between cambará x capivara; cambará x são joão; capivara x fortaleza; capivara $x$ são joão; and fortaleza $x$ são joão. Thus, for this study three management units were necessary to minimize the effects of genotypes $x$ environments interaction. Including selection for stability, adaptability, and both attributes simultaneously, there was a change between clones and the position of the clones in the order of selection. With the simultaneous selection for stability and adaptability (MHPRVG), the gains increased compared to the selection for predicted genotypic values among environments, especially; and there was an increase in gains compared to the commercial control. For example, the five best clones according to the criterion MHPRVG are 56\% higher than the commercial control for the characteristic productivity in volume.

Keywords: Adaptability; Stability; Genetic gain.

\section{INTRODUÇÃO}

A análise da interação genótipos $\mathrm{x}$ ambientes em Eucalyptus é fundamental para selecionar os melhores materiais genéticos com base no seu desempenho em diferentes condições edafoclimáticas de plantio (NUNES et al., 2002; ROCHA et al., 2005; ROSADO, et al., 2012). O desempenho de cultivares varia, normalmente, com a mudança de ambientes, de modo que um cultivar dificilmente é o melhor em todas as condições de cultivo (VENDUSCROLO et al., 2001), e isso significa, na prática, que ocorre interação genótipos x ambientes. Porém, na rotina da implantação florestal no Brasil, algumas vezes essa análise é negligenciada. Alguns poucos clones, que adquirem a confiança de determinada empresa florestal e de plantadores florestais da região (fomentados, pequenos e médios proprietários de terra), são plantados em toda a região de influência dessa empresa. Em geral, isso acontece porque o clone apresenta boa produtividade, é de boa propagação em viveiro e sua silvicultura é considerada fácil, ou seja, desenvolve bem nos primeiros dois anos, diminuindo custos de silvicultura. Porém, por muitas vezes, essa "região de influência" de determinada empresa abrange milhares de hectares e pode conter ambientes ou microclimas diferenciados, onde esses clones não apresentam desempenho tão estável, conforme o esperado.

Muitos autores consideram que a avaliação de genótipos, visando à identificação e recomendação de materiais genéticos superiores para as características de interesse em diferentes ambientes, é uma das etapas mais importantes de um programa de melhoramento (CRUZ; CARNEIRO, 2003; SILVA; DUARTE, 2006; RESENDE, 2007). Por consequência, essa também será uma das etapas mais importantes para o sucesso de um programa de silvicultura clonal em escala comercial, com o plantio de clones em diferentes ambientes produtivos (SANTOS et al., 2012).

O Estado do Rio Grande do Sul apresenta diferenças edafoclimáticas importantes, principalmente no que tange aos tipos de solo, em que as diferenças marcantes são a presença de solos encharcados, que favorecem a anoxia do sistema radicular (COSTA et al., 2009) e a presença de geadas em algumas regiões do Estado (TOSI; VÉLEZ-RODRIGUES, 1983). Em determinados anos, algumas regiões também podem sofrer com o déficit hídrico, entre os meses de dezembro e fevereiro. Assim, programas de melhoramento florestal e silvicultura clonal para o Estado do Rio Grande do Sul devem considerar como um dos principais fatores a serem considerados para o seu sucesso o bom entendimento da produtividade, adaptação e estabilidade dos materiais genéticos nas diferentes condições edafoclimáticas do Estado.

O termo adaptabilidade pode ser entendido como a capacidade de os genótipos responderem vantajosamente à melhoria do ambiente, enquanto a estabilidade se refere à capacidade de eles apresentarem comportamento altamente previsível em função das variações ambientais (CRUZ et al., 2004).

A seleção simultânea por produtividade, estabilidade e adaptabilidade, no contexto dos modelos mistos, pode ser realizada pelo método da Média Harmônica da Performance Relativa dos Valores Genéticos (MHPRVG) preditos. Esse método permite selecionar simultaneamente três atributos mencionados e apresenta as seguintes vantagens: (a) considera os efeitos genotípicos como aleatórios e, portanto, fornece estabilidade e adaptabilidade genotípica e não fenotípica; (b) permite lidar com desbalanceamento; (c) permite lidar com delineamentos não ortogonais; (d) permite 
lidar com heterogeneidade de variâncias; (e) permite considerar erros correlacionados dentro de locais; (f) fornece valores genéticos já descontados (penalizados) da instabilidade; (g) pode ser aplicado com qualquer número de ambientes; (h) permite considerar a estabilidade e adaptabilidade na seleção de indivíduos dentro de progênie; (i) não depende da estimação de outros parâmetros, tais quais coeficientes de regressão; (j) gera resultados na própria grandeza ou escala do caráter avaliado; e (1) permite computar o ganho genético com a seleção pelos três atributos simultaneamente (RESENDE, 2004; RESENDE, 2007).

Na última década, esse método tem sido usado com muito sucesso na análise de produtividade, estabilidade e adaptabilidade em diferentes culturas, como: cana-de-açúcar (BASTOS et al., 2007), feijão (CARBONELL et al., 2007), arroz (BORGES et al., 2009), seringueira (VERARDI et al., 2009), caju (MAIA et al., 2009), erva-mate (STURION; RESENDE, 2005) e pinus (MARTINEZ, 2010) e também em progênies de Eucalyptus (SILVA, 2008). Porém, são raros na literatura trabalhos que usam o método MHPRVG para a análise da interação genótipos x ambientes em clones de Eucalyptus.

Dessa maneira, o objetivo deste trabalho foi avaliar a presença de interação genótipos x ambientes, bem como selecionar clones para diferentes ambientes edafoclimáticos do Estado do Rio Grande do Sul, baseado na análise da produtividade, estabilidade e adaptabilidade.

\section{MATERIAL E MÉTODOS}

\section{1. Áreas de Estudo}

Os ensaios foram conduzidos nas áreas da empresa CMPC Celulose Riograndense, nos Municípios de Minas do Leão (Horto Florestal Cambará), Encruzilhada do Sul (Horto Florestal Capivara), Dom Feliciano (Horto Florestal Fortaleza) e Vila Nova do Sul (Horto Florestal São João), todos localizados no Estado do Rio Grande do Sul. Os experimentos foram plantados no espaçamento $3,5 \times 2,6$, cujos dados edafoclimáticos de cada local podem ser observados na Tabela 1 .

\subsection{Tomada de Dados}

Aos 3 anos de idade, foram mensurados o Diâmetro à Altura do Peito (dap) e a Altura Total $(\mathrm{Ht})$ das árvores dos experimentos. $\mathrm{O}$ dap foi mensurado com o auxílio de uma fita diamétrica e a altura, obtida com o uso do relascópio.
Para o cálculo do volume sem casca, foi utilizado o modelo de Leite et al. (1995), conforme apresentado a seguir.

$$
\begin{gathered}
\mathrm{V}=0,000048 * \operatorname{dap}^{1,720483 *} \text { altura }^{1,180736 *} \\
\mathrm{e}^{(-3,00555) *(\mathrm{t} x / \mathrm{dap}) *}\left\{1-(\mathrm{d} / \text { dap })^{1+0,228531 * \mathrm{~d}}\right\}+\mathrm{C} \\
\mathrm{R}^{2}=99,36
\end{gathered}
$$

em que dap: diâmetro a 1,3 m de altura; altura: altura total; $\mathrm{t} x=0$ para volume com casca e 1 para volume sem casca; d: diâmetro comercial superior; e $\epsilon=$ erro experimental.

\subsection{Delineamentos Estatísticos e Análise de Estabilidade eAdaptabilidade}

No ano 2007, foi implantada uma rede de testes clonais com 864 clones de Eucalyptus em quatro ambientes do Estado do Rio Grande do Sul. Como testemunha comparativa, foi utilizado o principal clone comercial empregado pela empresa (clone 32864).

Em cada ambiente foi estabelecido um experimento no delineamento de blocos ao acaso, com parcela de árvore única, com 30 repetições.

O modelo estatístico para análise dessa rede experimental em vários ambientes, considerando a tomada de uma observação por parcela, é dado por:

$$
\mathrm{y}=\mathrm{Xb}+\mathrm{Zg}+\mathrm{Wge}+\mathrm{e}
$$

em que:

$\mathrm{y}, \mathrm{b}, \mathrm{g}, \mathrm{ge}, \mathrm{e}=$ vetores de dados, de efeitos fixos (médias de blocos através dos locais), de efeitos genotípicos (aleatório), de efeitos da interação genótipos x ambientes (aleatório) e de erros aleatórios, respectivamente.

$\mathrm{X}, \mathrm{Z}$ e $\mathrm{W}=$ matrizes de incidência para $\mathrm{b}$, g e ge, respectivamente.

Distribuições e estruturas de médias e variâncias:

$$
E\left[\begin{array}{c}
y \\
g \\
g e \\
e
\end{array}\right]=\left[\begin{array}{c}
X b \\
0 \\
0 \\
0
\end{array}\right] ; \quad \operatorname{Var}\left[\begin{array}{c}
g \\
g e \\
e
\end{array}\right]=\left[\begin{array}{ccc}
I \sigma_{g}^{2} & 0 & 0 \\
0 & I \sigma_{g e}^{2} & 0 \\
0 & 0 & I \sigma_{e}^{2}
\end{array}\right]
$$

Equações de modelo misto:

$$
\left[\begin{array}{ccc}
X^{\prime} X & X^{\prime} Z & X^{\prime} W \\
Z^{\prime} X & Z^{\prime} Z+I \lambda_{1} & Z^{\prime} W \\
W^{\prime} X & W^{\prime} Z & W^{\prime} W+I \lambda_{2}
\end{array}\right]\left[\begin{array}{c}
\hat{b} \\
\hat{g} \\
\hat{g} e
\end{array}\right]=\left[\begin{array}{c}
X^{\prime} y \\
Z^{\prime} y \\
W^{\prime} y
\end{array}\right] \text {, em que }
$$

Revista Árvore, Viçosa-MG, v.39, n.1, p.81-91-2015 
Tabela 1 - Localização geográfica e condições edafoclimáticas de quatro ambientes do Estado do Rio Grande do Sul, utilizados para experimentação.

Table 1 - Geographical location and edaphoclimatic conditions of four environments of the State of Rio Grande do Sul, Brazil, used for experiments.

\begin{tabular}{|c|c|c|c|c|}
\hline & Cambará & Capivara & Fortaleza & São João \\
\hline \multirow[t]{2}{*}{ Coordenadas } & Latitude: & Latitude: & Latitude: & Latitude: \\
\hline & $30^{\circ} 11^{\prime} 09^{\prime \prime} \mathrm{S}$ & $30^{\circ} 27^{\prime} 19^{\prime \prime} \mathrm{S}$ & $30^{\circ} 29^{\prime} 45^{\prime \prime} \mathrm{S}$ & $30^{\circ} 14^{\prime} 46^{\prime \prime} \mathrm{S}$ \\
\hline \multirow[t]{2}{*}{ Geográficas } & Longitude & Longitude: & Longitude: & Longitude: \\
\hline & $52^{\circ} 00^{\prime} 10^{\prime \prime} \mathrm{W}$ & $52^{\circ} 39^{\prime} 53^{\prime \prime} \mathrm{W}$ & $52^{\circ} 19^{\prime} 35^{\prime \prime} \mathrm{W}$ & $53^{\circ} 49^{\prime} 7^{\prime \prime} \mathrm{W}$ \\
\hline Altitude (metros) & 141 & 250 & 378 & 301 \\
\hline Município & Minas do Leão & Encruzilhada do Sul & Dom Feliciano & Vila Nova do Sul \\
\hline Ocupação anterior & $\begin{array}{c}\text { Plantio de Eucalyptus } \\
\text { (Área de reforma) }\end{array}$ & Pastagem & Pastagem & Pastagem \\
\hline Tipo de Solo & $\begin{array}{c}\text { Argissolo Vermelho } \\
\text { distrófico } \\
\text { nitossólico- } \\
\text { textura argilosa }\end{array}$ & $\begin{array}{c}\text { Argissolo Vermelho } \\
\text { distrófico } \\
\text { abrúptico- } \\
\text { textura arenosa/argilosa }\end{array}$ & $\begin{array}{c}\text { Argissolo Vermelho } \\
\text { Amarelo } \\
\text { distrófico típico- } \\
\text { textura média/argilosa }\end{array}$ & $\begin{array}{l}\text { Argissolo Vermelho } \\
\text { distróficotípico - } \\
\text { textura média/argilosa }\end{array}$ \\
\hline Fertilidade & média a alta & média a alta & média a alta & média a baixa \\
\hline Profunidade do solo (mm) & profundo & médio & raso & profundo \\
\hline Relevo & plano suave ondulado & ondulado & ondulado & plano suave ondulado \\
\hline Mudança textural (perfil do solo) & leve & moderada & forte & leve \\
\hline Temperatura Média $\left({ }^{\circ} \mathrm{C}\right)$ & $17,5^{\circ} \mathrm{C}$ & $17,0^{\circ} \mathrm{C}$ & $16,0^{\circ} \mathrm{C}$ & $16,8^{\circ} \mathrm{C}$ \\
\hline Temperatura mínima absoluta $\left({ }^{\circ} \mathrm{C}\right)$ & $-0,9\left({ }^{\circ} \mathrm{C}\right)$ & $-0,6\left({ }^{\circ} \mathrm{C}\right)$ & $-1,7\left({ }^{\circ} \mathrm{C}\right)$ & $-0,0(\mathrm{oC})$ \\
\hline Temperatura máxima absoluta $\left({ }^{\circ} \mathrm{C}\right)$ & $32,3\left({ }^{\circ} \mathrm{C}\right)$ & $33,3\left({ }^{\circ} \mathrm{C}\right)$ & $30,7\left({ }^{\circ} \mathrm{C}\right)$ & $34,7\left({ }^{\circ} \mathrm{C}\right)$ \\
\hline Umidade relativa $(\%)$ & 77,6 & 74,8 & 80,6 & 75,6 \\
\hline Pluviosidade (mm) & 1.422 & 1.368 & 1.564 & 1.133 \\
\hline Velocidade do vento (metros/segundo) & 5,5 & 5,4 & 4,9 & 6,2 \\
\hline
\end{tabular}

$$
\lambda_{1}=\frac{\sigma_{e}^{2}}{\sigma_{g}^{2}}=\frac{1-h_{g}^{2}-c_{g e}^{2}}{h_{g}^{2}} ; \quad \lambda_{2}=\frac{\sigma_{e}^{2}}{\sigma_{g e}^{2}}=\frac{1-h_{g}^{2}-c_{g e}^{2}}{c_{g e}^{2}} .
$$

$h_{g}^{2}=\frac{\sigma_{g}^{2}}{\sigma_{g}^{2}+\sigma_{g e}^{2}+\sigma_{e}^{2}}=$ herdabilidade individual no sentido amplo de parcelas individuais no bloco;

$c_{g e}^{2}=\frac{\sigma_{g e}^{2}}{\sigma_{g}^{2}+\sigma_{g e}^{2}+\sigma_{e}^{2}}:$ coeficiente de determinação dos efeitos da interação genótipos $\mathrm{x}$ ambientes;

$\sigma_{g}^{2}=$ variância genotípica;

$\sigma_{g e}^{2}=$ variância da interação genótipos x ambientes;

$\sigma_{e}^{2}=$ variância residual entre parcelas; e $r_{g l o c}=\frac{\sigma_{c}^{2}}{\sigma_{g}^{2}+\sigma_{g e}^{2}}=\frac{h_{g}^{2}}{h_{g}^{2}+c_{g e}^{2}}$ : correlação genotípica dos materiais genéticos, através dos ambientes.
Estimadores de componentes de variância por REML via algoritmo EM:

$$
\begin{aligned}
& \hat{\sigma}_{e}^{2}=\left[\begin{array}{llll}
y^{\prime} y-\hat{b}^{\prime} & X^{\prime} y-\hat{g}^{\prime} & Z^{\prime} y-g \hat{e}^{\prime} & W^{\prime} y
\end{array}\right] /[N-r(x)] \\
& \left.\hat{\sigma}_{g}^{2}=\left[\hat{g}^{\prime} \hat{g}+\hat{\sigma}_{e}^{2} \operatorname{tr} C^{22}\right)\right] / q \\
& \hat{\sigma}_{g e}^{2}=\left[g \hat{e}^{\prime} g \hat{e}+\hat{\sigma}_{e}^{2} \operatorname{tr} C^{33}\right] / s
\end{aligned}
$$

em que:

$$
\begin{aligned}
& \mathrm{C}^{22} \text { e } \mathrm{C}^{33} \text { advém de: } \\
& C^{-1}=\left[\begin{array}{lll}
C_{11} & C_{12} & C_{13} \\
C_{21} & C_{22} & C_{23} \\
C_{31} & C_{32} & C_{33}
\end{array}\right]^{-1}=\left[\begin{array}{lll}
C^{11} & C^{12} & C^{13} \\
C^{21} & C^{22} & C^{23} \\
C^{31} & C^{32} & C^{33}
\end{array}\right]
\end{aligned}
$$

$\mathrm{C}=$ matriz dos coeficientes das equações de modelo misto;

$\operatorname{tr}=$ operador-traço matricial;

$\mathrm{r}(\mathrm{x})=$ posto da matriz $\mathrm{X}$; e

$\mathrm{N}, \mathrm{q}, \mathrm{s}=$ número total de dados, número de clones e número de combinações genótipos $\mathrm{x}$ ambientes, respectivamente. 
Nesse modelo, os valores genotípicos preditos livres da interação, considerando todos os locais, são dados por $\mathrm{u}+\mathrm{g}$, em que u é a média de todos os locais. Para cada local j, os valores genotípicos são preditos por $u_{j}+g+$ ge, em que $u_{j}$ é a média do local $j$.

A seleção conjunta por produtividade, estabilidade e adaptabilidade dos materiais genéticos foi baseada na estatística denominada média harmônica da performance relativa dos valores genéticos (MHPRVG) preditos, conforme descrito por Resende (2004). Todas as análises foram realizadas por meio do software Selegen-Reml/Blup.

Com os valores genéticos preditos, foram obtidas as correlações por ambiente, conjunto entre os ambientes e, ainda, a correlação entre os locais tomados 2 a 2 .

\section{RESULTADOS}

\subsection{Análises Genéticas e Estatísticas}

De acordo com a Tabela 2, em todas as características avaliadas as acurácias seletivas encontradas $(0,75$ a 0,98 ) foram sempre altas ou muito altas, conforme classificação de Resende e Duarte (2007), demonstrando boa qualidade experimental e segurança na seleção realizada nesses experimentos, para os caracteres avaliados. Verifica-se ainda, nessa tabela, a presença de variabilidade genética significativa entre os clones avaliados, conforme demonstrado pelas estimativas de herdabilidade e seus desvios-padrão (Tabela 2).

O coeficiente de variação genotípica dos caracteres observados neste estudo (CVgi) variou de 5,0\% para a característica $H t$ e $17,8 \%$ para a característica Volume. A presença de considerável variabilidade genética, como a encontrada neste estudo, indica a possibilidade de se praticar efetiva seleção entre os clones (RESENDE, 2007).

No geral, a correlação genotípica entre os ambientes $\left(\mathrm{r}_{\text {gloc }}\right)$ foi de baixa a moderada, para todos os caracteres avaliados (RESENDE; DUARTE, 2007), variando de 0,391 a 0,567 .

\subsection{Análises dos Ambientes Dois a Dois}

Na Tabela 3, pode-se observar a correlação genética do caráter produtividade em volume ( $\mathrm{m}^{3} /$ ha.ano), quando os locais são avaliados 2 a 2. Somente a correlação entre Cambará e Fortaleza $(0,67)$ pode ser considerada alta (RESENDE; DUARTE, 2007). Para as demais combinações, a correlação foi baixa, indicando a importância da seleção de clones específicos para esses ambientes.

As médias de produtividade em volume ( $\mathrm{m}^{3} /$ ha.ano) de cada local estudado, aos 3 anos de idade, foram

Tabela 2 - Estimativas de parâmetros genéticos (REML individual) dos caracteres diâmetro à altura do peito (dap), altura total (ht), volume ( $\mathrm{m}^{3} / \mathrm{ha}$.ano) e sobrevivência (\%) de clones de Eucalyptus, avaliados na análise conjunta entre os ambientes.

Table 2 - Estimates of genetic parameters (REML individual) of diameter at breast height (dbh), total height (ht), volume $\left(\mathrm{m}^{3} /\right.$ ha.year), and survival (\%) of Eucalyptus clones, evaluated in the joint analysis among environments.

\begin{tabular}{lcccc}
\hline & DAP $(\mathrm{cm})$ & HT $($ metros $)$ & Volume $\left(\mathrm{m}^{3} /\right.$ ha.ano) & Sobrevivência $(\%)$ \\
\hline $\mathrm{h}^{2}{ }_{\mathrm{g}}$ & $0,176 \pm 0,005$ & $0,070 \pm 0,003$ & $0,147 \pm 0,004$ & 0,387 \\
$\mathrm{~h}^{2}{ }_{\mathrm{mc}}$ & 0,441 & 0,222 & 0,145 & $0,024 \pm 0,002$ \\
$\mathrm{C}^{2}{ }_{\text {int }}$ & 0,134 & 0,104 & 0,860 & 0,037 \\
$\mathrm{Acgen}$ & 0,891 & 0,805 & 0,740 & 0,755 \\
$\mathrm{H}^{2}{ }_{\text {mg }}$ & 0,794 & 0,648 & 0,503 & 0,391 \\
$\mathrm{r}_{\text {gloc }}$ & 0,567 & 0,401 & 32,845 & 84,747 \\
Média geral & 13,266 & 14,726 & 17,891 & 6,418 \\
CVgi (\%) & 8,167 & 5,094 & 37,805 & 39,268 \\
CVe $(\%)$ & 15,710 & 16,501 &
\end{tabular}

$\mathrm{h}^{2} \mathrm{~g}$ : Coeficientes de herdabilidade individual no sentido amplo, livre da interação;

$\mathrm{h}^{2} \mathrm{mc}$ : herdabilidade da média de clone;

$\mathrm{C}^{2}$ : Coeficiente de determinação da interação genótipos $\mathrm{x}$ ambientes;

Acgen: Acurácia genética na seleção de clones:

$\mathrm{h}^{2}{ }_{\mathrm{mg}}$ : herdabilidade ajustada da média de genótipos, assumindo sobrevivência completa;

$\mathrm{r}_{\text {gloc }}$ : Correlação genotípica entre os ambientes;

Média geral: Média geral dos caracteres entre os diferentes ambientes;

CVgi (\%): Coeficiente de variação genotípica;

CVe (\%): Coeficiente de variação experimental; 
de 37,$3 ; 36,8 ; 32,1$; e 26,5 $\mathrm{m}^{3} /$ ha. ano, respectivamente nos locais São João, Cambará, Capivara e Fortaleza. A média geral dos quatro locais foi de $32,8 \mathrm{~m}^{3} /$ ha.ano. As produtividades nos locais São João e Cambará podem ser consideradas similares e melhores em relação ao site Capivara. Por sua vez, o site Capivara foi superior ao site Fortaleza.

\subsection{Ganhos Genéticos}

Na Tabela 4, pode-se observar o ordenamento dos 30 melhores clones da seleção conjunta entre os quatro ambientes, para a característica produtividade em volume ( $\mathrm{m}^{3} /$ ha.ano).

O ganho genético em relação à média geral do experimento, utilizando, por exemplo, os cinco melhores clones do ordenamento, é da ordem de $43,0 \%$, indicando boa possibilidade de ganho com a realização da seleção nessas condições. Porém, quando comparado com a testemunha do experimento (clone 32864), o ganho utilizando os mesmos cinco melhores clones diminui para $14,0 \%$. Isso indica menor possibilidade de ganho genético em relação à testemunha, em comparação com o ganho relativo à média geral de todos os clones dos experimentos.

\subsection{Estabilidade e Adaptabilidade}

Na Tabela 5 são apresentados os resultados sobre a estabilidade (MHVG), adaptabilidade (PRVG) e estabilidade e adaptabilidade, simultaneamente (MHPRVG), para a característica produtividade em volume ( $\mathrm{m}^{3} /$ ha.ano).

Verificou-se, por exemplo, que os cinco melhores clones com base nos critérios PRVG, MHVG e MHPRVG não coincidem totalmente com os cinco melhores clones

Tabela 3 - Correlação genética entre os ambientes avaliados dois a dois, para a característica produtividade em volume $\left(\mathrm{m}^{3} /\right.$ ha.ano $)$.

Table 3 - Genetic correlation between the environments evaluated two by two, for volume productivity $\left(\mathrm{m}^{3}\right)$ ha.year).

\begin{tabular}{lcccc}
\hline & Cambará & Capivara & Fortaleza & São João \\
\hline Cambará & - & 0,47 & 0,67 & 0,55 \\
Capivara & & - & 0,35 & 0,49 \\
Fortaleza & & & - & 0,48 \\
São João & & & & - \\
Volume & & 32,1 & 26,5 & 37,3 \\
$\left(\mathrm{~m}^{3} /\right.$ ha.ano $)$ & 36,8 & & & \\
\hline
\end{tabular}

pelo ordenamento de valores genotípicos preditos pela análise conjunta entre os ambientes (Tabela 4). A coincidência foi de $67 \%$ entre os cinco melhores clones, e houve inversão de ordem entre os coincidentes.

Comparando os ganhos obtidos com a MHPRVG em relação à testemunha (clone 32864), a superioridade média desses cinco genótipos foi de $29 \%$. Ao analisar esse ganho com o ganho predito no ordenamento de valores genotípicos da análise conjunta entre os ambientes (Tabela 4), também em relação à testemunha, concluiu-se que ele foi de $14 \%$.

Isso significa um ganho adicional de $15 \%$ em relação à testemunha, quando se utiliza a seleção simultânea por adaptabilidade e estabilidade dos valores genotípicos (MHPRVG). Segundo Resende (2007), isso ocorre porque, com a seleção simultânea com os novos materiais genéticos, se capitaliza o ganho com a interação média entre os ambientes, o que não acontece com o material genético usado como testemunha, pois eles estão com muitas repetições nos ensaios e sua herdabilidade média já tende a ser 1,0 em cada ensaio.

\section{DISCUSSÃO}

\subsection{Análises Genéticas e Estatísticas}

A presença de variabilidade encontrada permite inferir que há boa chance de sucesso na seleção para esses caracteres (MAIA et al., 2009) e deverá ser possível a obtenção de ganhos genéticos significativos.

Segundo Vencovsky e Barriga (1992), baixos valores de correlação entre os ambientes indicam a presença de interação genótipos x ambientes do tipo complexa, indicando a necessidade da seleção de clones específicos para os diferentes ambientes, visando à maximização dos ganhos genéticos esperados. Além disso, por esses resultados, justifica-se considerar na seleção desses clones suas adaptabilidades e estabilidades (RESENDE, 2007).

De acordo com Cruz e Regazzi (1994) e Lynch e Walsh (1998), a existência da interação está associada a dois fatores: o primeiro, denominado simples, é proporcionado pela diferença de variabilidade entre genótipos nos ambientes; e o segundo, denominado complexo, indica a inconsistência da superioridade de genótipos com a variação ambiental, ou seja, haverá genótipos com desempenho superior em um ambiente, 
mas não em outro, tornando mais difícil atingir os objetivos de seleção (RESENDE, 2007).

A melhor produtividade dos sites São João e Cambará é explicada pela sua melhor qualidade de solo, principalmente no que tange à boa profundidade $\mathrm{e}$ mudança textural (leve) dos solos desses ambientes (Tabela 1). Segundo Costa et al. (2009), no Estado do Rio Grande do Sul a produtividade florestal é fortemente afetada por algumas características dos solos, uma vez que solos rasos e com mudança textural de moderada a forte favorecem o alagamento de algumas áreas e a consequente anoxia do sistema radicular de Eucalyptus.
Outro fator que favorece a melhor produtividade desses ambientes, no que tange ao clima, é o relevo dessas áreas (plano suave ondulado), que são menos propícios à formação de geadas, em contraste com o relevo ondulado, que favorece a formação de geadas severas (STAHL et al., 2012). Esse tipo de relevo é encontrado nos sites Capivara e Fortaleza.

\subsection{Análise dos Ambientes 2 a 2}

Pode-se afirmar que, para Cambará e Fortaleza, um mesmo grupo de clones pode ser selecionado para plantio comercial nesses ambientes. Porém, nos sites

Tabela 4 - Ordenamento de clones por seus valores genotípicos e ganhos preditos para o caráter produtividade em volume ( $\mathrm{m}^{3} /$ ha.ano), na análise conjunta entre os ambientes.

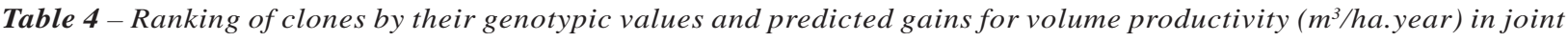
analysis among environments.

\begin{tabular}{|c|c|c|c|c|c|c|c|}
\hline $\begin{array}{l}\text { Posição } \\
\text { (ranking) }\end{array}$ & Genótipo & $\mathrm{g}$ & $\mathrm{u}+\mathrm{g}$ & Ganho & Nova média & $\begin{array}{l}\text { Ganho em relação } \\
\text { (média geral) }\end{array}$ & $\begin{array}{c}\text { Ganho em relação } \\
\text { (testemunha) }\end{array}$ \\
\hline 1 & 39654 & 14,3 & 47,2 & 14,3 & 47,2 & $43,6 \%$ & $14,7 \%$ \\
\hline 2 & 39569 & 14,3 & 47,1 & 14,3 & 47,1 & $43,5 \%$ & $14,6 \%$ \\
\hline 3 & 39416 & 13,7 & 46,6 & 14,1 & 47,0 & $43,0 \%$ & $14,1 \%$ \\
\hline 4 & 39659 & 13,5 & 46,4 & 14,0 & 46,8 & $42,5 \%$ & $13,8 \%$ \\
\hline 5 & 39030 & 12,4 & 45,3 & 13,7 & 46,5 & $41,6 \%$ & $13,1 \%$ \\
\hline 6 & 39045 & 12,4 & 45,2 & 13,4 & 46,3 & $40,9 \%$ & $12,5 \%$ \\
\hline 7 & 39095 & 12,1 & 45,0 & 13,3 & 46,1 & $40,4 \%$ & $12,1 \%$ \\
\hline 8 & 39438 & 12,0 & 44,8 & 13,1 & 46,0 & $39,9 \%$ & $11,7 \%$ \\
\hline 9 & 39032 & 12,0 & 44,8 & 13,0 & 45,8 & $39,5 \%$ & $11,3 \%$ \\
\hline 10 & 39606 & 11,7 & 44,5 & 12,9 & 45,7 & $39,1 \%$ & $11,1 \%$ \\
\hline 11 & 38885 & 11,6 & 44,4 & 12,7 & 45,6 & $38,8 \%$ & $10,8 \%$ \\
\hline 12 & 38903 & 11,5 & 44,3 & 12,6 & 45,5 & $38,5 \%$ & $10,5 \%$ \\
\hline 13 & 39789 & 11,3 & 44,1 & 12,5 & 45,4 & $38,1 \%$ & $10,3 \%$ \\
\hline 14 & 39320 & 11,0 & 43,8 & 12,4 & 45,3 & $37,8 \%$ & $10,0 \%$ \\
\hline 15 & 39706 & 10,6 & 43,5 & 12,3 & 45,1 & $37,4 \%$ & $9,7 \%$ \\
\hline 16 & 39425 & 10,6 & 43,4 & 12,2 & 45,0 & $37,1 \%$ & $9,5 \%$ \\
\hline 17 & 39407 & 10,6 & 43,4 & 12,1 & 44,9 & $36,8 \%$ & $9,2 \%$ \\
\hline 18 & 6808 & 10,2 & 43,1 & 12,0 & 44,8 & $36,5 \%$ & $9,0 \%$ \\
\hline 19 & 39543 & 10,2 & 43,0 & 11,9 & 44,7 & $36,2 \%$ & $8,7 \%$ \\
\hline 20 & 39510 & 9,9 & 42,7 & 11,8 & 44,6 & $35,9 \%$ & $8,5 \%$ \\
\hline 21 & 39018 & 9,8 & 42,7 & 11,7 & 44,6 & $35,6 \%$ & $8,3 \%$ \\
\hline 22 & 39427 & 9,6 & 42,5 & 11,6 & 44,4 & $35,3 \%$ & $8,0 \%$ \\
\hline 23 & 39246 & 9,6 & 42,4 & 11,5 & 44,4 & $35,1 \%$ & $7,8 \%$ \\
\hline 24 & 38935 & 9,4 & 42,2 & 11,4 & 44,3 & $34,7 \%$ & $7,6 \%$ \\
\hline 25 & 39423 & 9,4 & 42,2 & 11,3 & 44,2 & $34,5 \%$ & $7,4 \%$ \\
\hline 26 & 38904 & 9,2 & 42,1 & 11,3 & 44,1 & $34,3 \%$ & $7,2 \%$ \\
\hline 27 & 39665 & 9,2 & 42,0 & 11,2 & 44,0 & $34,1 \%$ & $7,0 \%$ \\
\hline 28 & 39410 & 9,1 & 42,0 & 11,1 & 44,0 & $33,8 \%$ & $6,8 \%$ \\
\hline 29 & 6815 & 8,9 & 41,7 & 11,0 & 43,9 & $33,6 \%$ & $6,7 \%$ \\
\hline 30 & 38973 & 8,8 & 41,6 & 11,0 & 43,8 & $33,4 \%$ & $6,5 \%$ \\
\hline$\cdot$ & . & . & . & . & . & . & . \\
\hline \multirow[t]{2}{*}{94} & 32864(testemunha) & 5,9 & 38,8 & 8,3 & 41,1 & $25,3 \%$ & - \\
\hline & & & & Média geral & 32,8 & & \\
\hline
\end{tabular}


Tabela 5 - Ordenamento da estabilidade de valores genéticos (MHVG), adaptabilidade de valores genéticos (PRVG) e estabilidade e adaptabilidade simultaneamente (MHPRVG) para o caráter produtividade volume $\left(\mathrm{m}^{3} / \mathrm{ha}\right.$.ano).

Table 5 - Ranking of stability of genetic values (MHVG), adaptability of genetic values (PRVG) and stability and adaptability simultaneously (MHPRVG), for volume productivity ( $\mathrm{m}^{3} /$ ha.year).

\begin{tabular}{|c|c|c|c|c|c|c|c|c|}
\hline \multirow{2}{*}{ Ranking (Posição) } & \multicolumn{2}{|c|}{ Estabilidade } & \multicolumn{3}{|c|}{ Adaptabilidade } & \multicolumn{3}{|c|}{ Estabilidade e Adaptabilidade } \\
\hline & Genótipo & MHVG & Genótipo & PRVG & PRVG*MG & Genótipo & MHPRVG & MHPRVG*MG \\
\hline 1 & 6808 & 57,0 & $\underline{39654}$ & 1,633 & 53,6 & $\underline{39654}$ & 1,598 & 52,5 \\
\hline 2 & 6815 & 54,2 & $\underline{39416}$ & 1,578 & 51,8 & $\underline{39416}$ & 1,578 & 51,8 \\
\hline 3 & $\underline{39654}$ & 50,4 & $\underline{39569}$ & 1,575 & 51,7 & 6808 & 1,555 & 51,1 \\
\hline 4 & 39420 & 50,2 & 6808 & 1,555 & 51,1 & $\underline{39569}$ & 1,550 & 50,9 \\
\hline 5 & $\underline{39030}$ & 50,1 & $\underline{39659}$ & 1,535 & 50,4 & $\underline{39659}$ & 1,534 & 50,4 \\
\hline 6 & 39407 & 50,1 & 39543 & 1,483 & 48,7 & 39543 & 1,483 & 48,7 \\
\hline 7 & 39659 & 49,5 & 39912 & 1,483 & 48,7 & 39912 & 1,483 & 48,7 \\
\hline 8 & 39416 & 48,9 & 6815 & 1,480 & 48,6 & 6815 & 1,480 & 48,6 \\
\hline 9 & 39468 & 48,1 & 39606 & 1,474 & 48,4 & 38934 & 1,464 & 48,1 \\
\hline 10 & 39425 & 47,5 & 39407 & 1,467 & 48,2 & 39407 & 1,459 & 47,9 \\
\hline 11 & 39032 & 47,3 & 39030 & 1,466 & 48,2 & 39606 & 1,458 & 47,9 \\
\hline 12 & 39569 & 47,3 & 39032 & 1,466 & 48,1 & 39032 & 1,454 & 47,8 \\
\hline 13 & 39912 & 47,0 & 38934 & 1,464 & 48,1 & 39095 & 1,447 & 47,5 \\
\hline 14 & 39565 & 46,7 & 39095 & 1,458 & 47,9 & 11511 & 1,447 & 47,5 \\
\hline 15 & 39095 & 46,6 & 39045 & 1,457 & 47,9 & 39438 & 1,444 & 47,4 \\
\hline 16 & 39396 & 46,6 & 38885 & 1,454 & 47,7 & 39030 & 1,439 & 47,3 \\
\hline 17 & 39038 & 46,5 & 39510 & 1,449 & 47,6 & 39510 & 1,439 & 47,3 \\
\hline 18 & 39438 & 46,2 & 39438 & 1,448 & 47,5 & 39045 & 1,435 & 47,1 \\
\hline 19 & 38903 & 46,2 & 11511 & 1,447 & 47,5 & 38903 & 1,433 & 47,1 \\
\hline 20 & 38885 & 46,0 & 39706 & 1,444 & 47,4 & 38885 & 1,429 & 46,9 \\
\hline 21 & 38909 & 46,0 & 38903 & 1,437 & 47,2 & 38975 & 1,426 & 46,8 \\
\hline 22 & 39045 & 45,9 & 38975 & 1,426 & 46,8 & 36190 & 1,414 & 46,5 \\
\hline 23 & 39335 & 45,6 & 36190 & 1,414 & 46,5 & 39410 & 1,402 & 46,0 \\
\hline 24 & 39543 & 45,4 & 39410 & 1,412 & 46,4 & 39789 & 1,391 & 45,7 \\
\hline 25 & 39406 & 45,4 & 39789 & 1,411 & 46,4 & 38935 & 1,388 & 45,6 \\
\hline 26 & 39001 & 45,3 & 39246 & 1,411 & 46,3 & 39425 & 1,372 & 45,1 \\
\hline 27 & 32949 & 45,1 & 39320 & 1,406 & 46,2 & 39246 & 1,371 & 45,0 \\
\hline 28 & 39706 & 45,1 & 39425 & 1,390 & 45,6 & 39706 & 1,366 & 44,9 \\
\hline 29 & 39487 & 44,9 & 38935 & 1,390 & 45,6 & 39878 & 1,359 & 44,6 \\
\hline 30 & 36190 & 44,8 & 39018 & 1,359 & 44,6 & 39420 & 1,357 & 44,6 \\
\hline & . & . & . & . & & . & . & . \\
\hline \multirow{4}{*}{128} & 32864 & 38,7 & 32864 & 1,22 & 40,0 & 32864 & 1,21 & 39,7 \\
\hline & & & & & & & Média geral & 32,8 \\
\hline & & & \multicolumn{5}{|c|}{ Ganho genético em relação à média do experimento } & $56 \%$ \\
\hline & & & \multicolumn{5}{|c|}{ Ganho genético em relação à média da testemunha } & $29 \%$ \\
\hline
\end{tabular}

Obs: Clones sublinhados são os cinco melhores do ordenamento de valores genotípicos preditos, na seleção conjunta entre os ambientes, presentes também no ordenamento da seleção para estabilidade e produtividade $(10$ em $15,67 \%)$.

Capivara e São João, os clones que irão proporcionar os melhores ganhos genéticos não serão os mesmos.

Com base nesses resultados, pode-se afirmar que o Programa de Melhoramento Genético da Empresa, visando à produção de indivíduos para instalação de testes clonais e posterior seleção para plantios comerciais, deverá levar em consideração esses três diferentes ambientes (Cambará-Fortaleza, São João,
Capivara) e a resposta diferenciada do comportamento dos clones entre esses ambientes. Além disso, segundo Kang et al. (2006), quando são encontradas baixas correlações genéticas entre os ambientes, é importante evoluir para análises de adaptação e estabilidade, que proporcionam uma análise mais refinada do comportamento dos cultivares nos diferentes ambientes (RESENDE, 2007). 
Em termos do melhoramento, dependendo do que se pretende obter, a ocorrência de baixa correlação genética entre os ambientes nem sempre é desejável, existindo algumas opções para atenuar os efeitos da interação, como: (i) identificar genótipos específicos para cada ambiente; (ii) promover subdivisões de uma área heterogênea em sub-regiões mais uniformes, de modo que os genótipos não interajam significativamente com os ambientes; e (iii) identificar genótipos com maior estabilidade fenotípica (VENCOVSKY; BARRIGA, 1992). Esses mesmos autores relataram que a opção (ii) geralmente é a mais utilizada para minimizar os efeitos da interação.

Essa informação vai ao encontro do relatado por Resende (2005), que recomendou que uma das principais alternativas para esse tipo de situação é a estratificação dos locais de plantio, o que permite a definição de "zonas de melhoramento". Dentro dessas zonas, a interação genótipos x ambientes será desprezível, e entre elas a interação não será problemática para o melhorista. Assim, cada zona de melhoramento demanda um programa de melhoramento específico, e o número dessas zonas indicará o número mínimo de populações de melhoramento a serem empregadas pelo melhorista. Esse mesmo raciocínio pode ser utilizado para o número de "unidades de produção", em que é necessário o estabelecimento de testes clonais e seleção de clones específicos para plantios comerciais.

\subsection{Ganhos Genéticos}

Apesar de a testemunha, representada pelo clone 32864 , estar na $94^{\mathrm{a}}$ posição no ordenamento para produtividade em volume ( $\mathrm{m}^{3} /$ ha.ano), a sua diferença para os melhores clones dos experimentos não é grande. A comparação dos ganhos genotípicos preditos em relação à testemunha comercial plantada pela empresa é fundamental, uma vez que o objetivo de um programa de melhoramento é sempre melhorar a média dos materiais genéticos (clones) plantados comercialmente na atualidade e não somente melhorar a média da população ao longo do tempo.

Isso indica que o principal clone atualmente utilizado pela empresa apresenta bom desempenho na média dos ambientes, podendo ser considerado um clone plástico e razoavelmente adaptado às diferentes condições edafoclimáticas de plantio da empresa.

Com essa constatação, torna-se um desafio importante desenvolver materiais genéticos e critérios de seleção, que maximizem o ganho genético dos novos materiais desenvolvidos sobre a média da testemunha comercial.

\subsection{Estabilidade e Adaptabilidade}

A não coincidência no ordenamento dos melhores clones na análise de estabilidade e adaptabilidade, com o ordenamento por valores genotípicos pela análise conjunta entre os ambientes, demonstra que a utilização desses novos atributos ou critérios de seleção propicia refinamento na seleção (RESENDE, 2007). Os cinco melhores genótipos (39654, 39416, 6808, 39569 e 39659) pelo critério MHPRVG foram superiores, em média, 56\% sobre a média geral dos quatro ambientes $\left(32,8 \mathrm{~m}^{3} /\right.$ ha.ano). De acordo com Anputhas et al. (2011), a recomendação de cultivares com ampla adaptabilidade e estabilidade é essencial para regiões com diferentes ambientes produtivos, ou com estações climáticas marcantes. Essas duas condições são encontradas no Estado Rio Grande do Sul.

Os valores apresentados para MHPRVG foram computados, já penalizando os genótipos pela instabilidade através dos locais e, ao mesmo tempo, capitalizando a capacidade de resposta (adaptabilidade) à melhoria do ambiente (RESENDE, 2007; MAIA, 2009).

Conforme comentado anteriormente, o objetivo de um programa de desenvolvimento de clones para plantios comerciais é sempre superar o clone plantado operacionalmente na atualidade (testemunha). Dessa maneira, pode-se considerar que, para a situação estudada, a seleção considerando a adaptabilidade e estabilidade simultaneamente proporcionou ganhos significativamente superiores $(15 \%)$ e deve ser utilizada para a recomendação de novos clones dentro do Programa de Melhoramento Genético da Empresa.

\section{CONCLUSÕES}

Com base nos objetivos definidos neste trabalho, buscando avaliar a presença de interação genótipos $\mathrm{x}$ ambientes em quatro ambientes de cultivo de Eucalyptus no Rio Grande do Sul e, ainda, verificar a adaptabilidade de diferentes clones de híbridos de Eucalyptus a esses ambientes, conclui-se que há interação genótipos $\mathrm{x}$ ambientes do tipo complexo nessa região.

Os ambientes Cambará e Fortaleza apresentam alta correlação genética entre si, porém foi observada baixa correlação genética na combinação desses ambientes

Revista Árvore, Viçosa-MG, v.39, n.1, p.81-91-2015

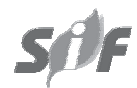


com os ambientes Capivara e São João. Dessa maneira, é importante que esses diferentes ambientes produtivos sejam considerados no Programa de Melhoramento Florestal da Empresa, bem como três "unidades de manejo" para recomendação de clones específicos para cada uma delas devem ser observadas (CambaráFortaleza, Capivara e São João).

Os cinco melhores genótipos $(39654,39416,6808$, 39569 e 39659) pelo critério MHPRVG foram superiores, em média, $56 \%$ sobre a média geral dos quatro ambientes e devem ser avaliados como alternativa para plantio comercial, em relação ao clone atualmente utilizado nessa região.

\section{AGRADECIMENTOS}

A empresa Klabin S.A., pela oportunidade inicial de realizar o doutorado que deu origem a esse trabalho e a CMPC Celulose Riograndense pelo apoio para a defesa da tese e publicação desse artigo.

\section{REFERÊNCIAS}

ANPUTHAS M.; SAMITA, S.; ABEYSIRIWARDENA, D.S. Stability and adaptability analysis of rice cultivars using environment-centered yield in two-way ANOVA model. Communications in Biometry and Crop Science, v.6, n.2, p.80-86, 2011.

BASTOS, I. T.; BARBOSA, M.H.P.; RESENDE, M.D.V.; PETERNELLI, L.A.; SILVEIRA, L.C.I.; DONDA, L. R.; FORTUNATO, A.A.; COSTA, P.M.A.C.; FIGUEIREDO, I.C.R. Avaliação da interação genótipo x ambiente em cana-de-açúcar via modelos mistos. Pesquisa Agropecuária Tropical, v.37, n.4, p.195-203, 2007.

BORGES, V.; SOARES, A.A.; RESENDE, M.D.V.; REIS, M.S.; CORNÉLIO, V.M.O.; SOARES, P.C. Progresso genético do programa de melhoramento de arroz de terras altas de Minas gerais utilizando modelos mistos. Revista Brasileira de Biometria, v.27, n.3, p.478490, 2009.

CARBONELL, S.A.M.; AZEVEDO FILHO, J.A.A.; DIAS, L.A.S.; GONÇALVES, C.; ANTONIO, C.B. Estabilidade de cultivares e linhagens de feijoeiro em diferentes ambientes no estado de São Paulo. Bragantia, v.66, n.2. p.193-201, 2007.

Revista Árvore, Viçosa-MG, v.39, n.1, p.81-91, 2015
COSTA, A.M.; CURI, N.; ARAÚJO, E.F.; MARQUES, J.J.; MENEZES, M.D. Avaliação do risco de anoxia para o cultivo do eucalipto no Rio Grande do Sul utilizando-se levantamento de solos. Scientia Forestalis, v.37, n.84, p.367375, 2009.

CRUZ, C. D.; REGAZZI, A. J.; CARNEIRO, P. C. S. Modelos biométricos aplicados ao melhoramento genético. Viçosa, MG, Universidade Federal de Viçosa, 2004. p.171-201. v.1.

CRUZ, C. D.; REGAZZI, A. J. Modelos

biométricos aplicados ao

melhoramento genético. 2.ed. Viçosa, MG, Universidade Federal de Viçosa, 1994. 390p.

CRUZ, C. D.; CARNEIRO, P. C. S. Modelos biométricos aplicados ao melhoramento genético. Viçosa, MG, Universidade Federal de Viçosa, 2003. v.2.

LEITE, H. G.; GUIMARÃES, D. P.; CAMPOS, J. C. C. Descrição e emprego de um modelo para estimar múltiplos volumes de árvores. Revista Árvore, v.19, n.1, p.75-79, 1995.

LYNCH, M. C.; WALSH, B. Genetics and analysis of quantitative traits.

Sunderland: Sinauer Associates, 1998. 980p.

KANG, M. S.; AGGARWAL, V. D.; CHIRWA, R. M. Adaptability and stability of bean cultivars as determined via yield-stability statistic and GGE biplot analysis. Journal of Crop

Improvement, v. 15, p.97-120, 2006.

MAIA, M.C.C; RESENDE, M.D.V; PAIVA, J.R; CAVALCANTI, J.J.V; BARROS, L.M. Seleção simultânea para produção, adaptabilidade e estabilidade genotípicas em clones de cajueiro, via modelos mistos. Pesquisa Agropecuária Tropical, v. 39, n. 1, p. 43-50, 2009.

MARtineZ, D. T. Avaliação genética sob heterogeneidade de variância residual dentro de tratamentos. 2010. 64f. Tese (Doutorado em Engenharia Florestal) Universidade Federal do Paraná, Curitiba, 2010.

NUNES, G. H. S.; RESENDE, G. D. S. P.; RAMALHO, M. A. P.; SANTOS, J. B. Implicações 
da interação genótipo x ambientes na seleção de clones de eucalipto. Cerne, v.8, n.1. p.49-58, 2002.

RESENDE, M. D. V.; DUARTE, J. B. Precisão e controle de qualidade em experimentos de avaliação de cultivares. Pesquisa

Agropecuária Tropical, v. 37, n. 3, p. 182$194,2007$.

RESENDE, M. D. V. Novas abordagens estatísticas na análise de experimentos e campo. Colombo: Embrapa Florestas, 2004. 60p. (Embrapa Florestas Documentos, 100).

RESENDE, M. D. V. Melhoramento de Essências Florestais. In: BORÉM, A. Melhoramento de espécies cultivadas. Viçosa, MG,

Universidade Federal de Viçosa, 2005. 717-780.

RESENDE, M. D. V. Matemática e estatística na análise de experimentos e no melhoramento genético. Colombo: Embrapa Florestas, 2007. 562p.

ROCHA, R.B.; MURO-ABAD, J.I.; ARAÚJO, E.F.; CRUZ, C.D. Avaliação do método centróide para estudo da adaptabilidade ao ambiente de clones de Eucalyptus grandis. Ciência Florestal, v.15, n.3, p.255-266, 2005

ROSADO, A. M. Seleção simultânea de clones de eucalipto de acordo com produtividade, estabilidade e adaptabilidade. Pesquisa Agropecuária Brasileira, v.47, n.7, p.964971,2012

SANTOS, G.A.; CABEL, S.R.; ASSIS, T.F.; FIER, I.S.N.; MENDES, C.J.; PUCCI, J.A.L. Potencial da silvicultura clonal de Eucalyptus benthamii para o sul do Brasil. In: SILVA, L. D.; HIGA, A. R.; SANTOS, G. A. (Coord.) Silvicultura e melhoramento genético de Eucalyptus benthamii. Curitiba: FUPEF, 2012. p.77-103.

SILVA, L. D. Melhoramento genético de Eucalyptus benthamii Maiden et Cambage visando a produção de madeira serrada em áreas de ocorrência de geadas severas. 2008. 275p. Tese (Doutorado em Engenharia Florestal) - Universidade Federal do Paraná, Curitiba, 2008.

SILVA, W. C. J.; DUARTE, J. B. Métodos estatísticos para estudo de adaptabilidade e estabilidade fenotípica em soja. Pesquisa agropecuária brasileira, Brasília, v.41, n.1, p.23-30, jan. 2006.

STAHL. J. Silvicultura de Eucalyptus benthamii na Klabin S.A. In: SILVA, L. D.; HIGA, A. R.; SANTOS, G. A. (Coord.) Silvicultura e melhoramento genético de Eucalyptus benthamii. Curitiba: FUPEF, 2012. p.105-121.

STURION, J. A.; RESENDE, M. D. V. Seleção de progênies de erva-mate (Ilex paraguariensis St. Hil.) para produtividade, estabilidade e adaptabilidade temporal de massa foliar. Boletim de Pesquisa Florestal, n.50, p.37-51, 2005.

TOSI, J. A.; VÉLEZ-RODRIGUEZ, L. L. Provisional ecological map of the republic of Brazil. San Juán: Institute of Tropical Forestry, 1983.

VENCOVSKY, R.; BARRIGA, P. Genética biométrica no fitomelhoramento. Ribeirão Preto: Sociedade Brasileira de Genética, 1992. 486p.

VENDRUSCROLO, E.C.G.; SCAPIM, C.A.; PACHECO, C.A.P.; OLIVEIRA, V.R.; BRACCINI, A.L.; GONÇALVES-VIDIGAL, M.C.

Adaptabilidade e estabilidade de cultivares de milho-pipoca na região centro-sul do Brasil. Pesquisa Agropecuária Brasileira, v.36, n.1, p.123-130, 2001.

VERARDI, C.K; RESENDE, M.D.V; COSTA, R.B; GONÇALVEZ, P.S. Adaptabilidade e estabilidade da produção de borracha e seleção em progênies de seringueira. Pesquisa Agropecuária Brasileira, v.44, n.10, p.1277-1282, 2009. 